\title{
Review of: "Comparing the cost of non-metastatic breast cancer care in a low-income vs a high-income country: A plea for an optimal allocation of health resources in Sub-Saharan Africa"
}

\author{
Amani Mori
}

Potential competing interests: The author(s) declared that no potential competing interests exist.

This study was published as a short communication in the journal of The Breast, and compared differences in the provider costs of managing non-metastatic breast cancer in Mozambiques and Portugal. Mozambique is a low-income country located in sub-Saharan Africa while Portugal is a high-income country. The study followed a cohort of women newly diagnosed with stage I-III Breast cancers for a period of three years. The authors estimated the median costs of 2,888 in Mozambique versus 18,533 Int\$ in Portugal. The study is important because it adds useful information required to inform resource allocation decisions as well as informing economic evaluation studies.

The paper has several limitations and needs to be interpreted with care.

First, it makes unwarranted comparisons, and the tone implies that Mozambique should devote the same amount of health care resources as Portugal to manage non-metastatic breast cancer. The authors could have instead of comparing the costs between the two countries conduct a scenario analysis [1] for Mozambique to compare the existing care provided (poor quality care) and a hypothetical best scenario reflecting optimal resource allocation. This could have answered the question "what additional resources are required to provide the needed standard of care for non-metastatic breast cancer in the context of Mozambique".

Second, the authors are not sufficiently transparent on how they arrived at the reported unit costs. There are so many issues that can be discussed here.

- Which type of costing method was used (bottom-up versus top-down), and whether the same method was applied in the two countries. They wrote that, in Portugal healthcare costs came from clinical and administrative records [2] while in Mozambique, according to the paper the healthcare costs were given by the Maputo Central Hospital and/or retrieved from the World Health Organization-CHOICE estimates [1]. It is obvious that the quality of data was good in Portugal but not in Mozambique, which could have introduced huge differences. In low-income countries it is much easier to underestimate resource use due to poor documentation. 
- Which type of costs were included in terms of capital versus recurrent cost items. Healthcare resources are extremely scarce in low-income countries; hence, there are fierce intersectoral competitions as opposed to high-income countries. As a result, hospitals in low-income countries are usually understaffed, medicines are frequently out of stock, diagnostic equipment may be absent or outdated etc. The authors have touched upon some of these issues when they say "Nonetheless, there were occasional treatment interruptions/adjustments in Mozambique due to drug supply shortages. Radiotherapy, trastuzumab and aromatase inhibitors were not available at the Maputo Central Hospital, but some Mozambican patients (6\%) received radiotherapy outside the country" [3]. But what about the healthcare personnel, type and size of buildings, diagnostic equipment etc?. If capital costs were included, were they discounted? At what rate? And was it the same for both countries?

- There is an issue of survival rates, which is not discussed in the paper and how it affected the resource use. One would expect that since the quality of care in Mozambique was sub-optimal then survival rates were lower, and hence reducing resource use and the median breast cancer costs over the three-year follow-up period.

- How different are the healthcare systems between the countries? Even though it is mentioned that both countries have universal health coverage, but the benefit package could be very different, which would have affected the costs. We saw that Radiotherapy, trastuzumab and aromatase inhibitors were not available at the Maputo Central Hospital, forcing some patients to seek it outside the country [3]".

Lastly, the authors should have added a limitation section to contextualize their findings. It was important to describe the weaknesses that are inherent in their paper, which I believe would have added value and helped the readers to understand. In this type of paper it was important to discussed issues of transferability of cost information and sample size as to whether their cost-estimates are representative for Mozambique and or Portugal.

1. Simoens S. Health economic assessment: a methodological primer. Int J Environ Res Public Health. 2009; 6(12):2950-66. doi: 10.3390/ijerph6122950.

1. Brandão M, Morais S, Lopes-Conceição L, Fontes F, Araújo N, Dias T, Pereira D, Borges M, Pereira S, Lunet $\mathrm{N}$. Healthcare use and costs in early breast cancer: a patient-level data analysis according to stage and breast cancer subtype. ESMO Open. 2020 Nov;5(6):e000984. doi: 10.1136/esmoopen-2020000984.

1. Brandão M, Morais S, Guisseve A, Bata G, Borges M, Tulsidás S, Pereira S, Carrilho C, Lunet N. Comparing the cost of non-metastatic breast cancer care in a low-income vs a high-income country: A plea for an optimal allocation of health resources in Sub-Saharan Africa. Breast. 2021 Jun;57:1-4. doi: 
10.1016/j.breast.2021.02.010. 